\title{
openheart Right atrium and cryptogenic ischaemic stroke in the young: a case-control study
}

\author{
Pauli Pöyhönen (D) , ${ }^{1}$ Jouni Kuusisto, ${ }^{1}$ Jani Pirinen, ${ }^{2,3}$ Heli Räty, ${ }^{2}$ Lauri Lehmonen, ${ }^{4}$ \\ Riitta Paakkanen, ${ }^{1}$ Nicolas Martinez-Majander, ${ }^{5}$ Eva Gerdts, ${ }^{6}$ Jukka Putaala, ${ }^{5}$ \\ Juha Sinisalo, ${ }^{1}$ Vesa Järvinen ${ }^{2}$
}

\begin{abstract}
- Additional supplemental material is published online only. To view, please visit the journal online (http://dx.doi.org/10.
\end{abstract} 1136/openhrt-2021-001596).

To cite: Pöyhönen P, Kuusisto J, Pirinen $\mathrm{J}$, et al. Right atrium and cryptogenic ischaemic stroke in the young: a case-control study. Open Heart 2021;8:e001596. doi:10.1136/ openhrt-2021-001596

Received 22 January 2021 Revised 13 March 2021 Accepted 3 May 2021

\section{Check for updates}

(C) Author(s) (or their employer(s)) 2021. Re-use permitted under CC BY-NC. No commercial re-use. See rights and permissions. Published by BMJ.

${ }^{1}$ Heart and Lung Center, Helsinki University Hospital and University of Helsinki, Helsinki, Finland

${ }^{2}$ Department of Clinical Physiology and Nuclear Medicine, HUS Medical Imaging Center, Helsinki, Finland ${ }^{3}$ Internal Medicine, HUS Porvoo Hospital Area, Porvoo, Finland ${ }^{4}$ Radiology, HUS Medical Imaging Center, Helsink University Hospital and University of Helsinki, Helsinki Finland

${ }^{5}$ Neurology, Helsinki University Hospital and University of Helsinki, Helsinki, Finland ${ }^{6}$ Department of Clinical Science, University of Bergen, Bergen, Norway

Correspondence to Dr Pauli Pöyhönen; pauli. poyhonen@helsinki.fi

\section{ABSTRACT}

Background Recent studies suggest left atrial (LA) dysfunction in cryptogenic stroke. We studied the dynamics of right atrium (RA) and right atrial appendage (RAA) in young adults with cryptogenic stroke. We hypothesised that bi-atrial dysfunction and blood stagnation might contribute to thrombosis formation in patients with patent foramen ovale (PF0), as deep venous thrombosis is detected only in the minority of patients. Methods Thirty patients (aged 18-49) with a first-ever cryptogenic stroke and 30 age-matched and sex-matched stroke-free controls underwent cardiac magnetic resonance (CMR) imaging. An approach to estimate the RAA volume was developed, using crista terminalis and pectinate muscles as anatomical landmarks. Atrial expansion indices were calculated as (maximal volume minimal volume) $\times 100 \% /$ minimal volume. Total pulmonary to systemic blood flow ratio (Qp/Qs) was based on phase contrast CMR. Right-to-left shunt (RLS) was evaluated with transoesophageal echocardiography in 29 patients and transcranial Doppler in 30 controls, moderate-to-severe RLS considered as clinically significant.

Results We found that RA and RAA volumes were similar between patients and controls. Also, RA expansion index was similar, but RAA $(95.6 \% \pm 21.6 \%$ vs $108.7 \% \pm 25.8 \%$, $p=0.026)$ and $L A(126.2 \% \pm 28 \%$ vs $144.9 \% \pm 36.3 \%$, $\mathrm{p}=0.023$ ) expansion indices were lower in patients compared with controls. Seven (24\%) of 29 patients had an RLS compared with $1(3 \%)$ of 30 controls ( $p=0.012)$. Among 59 study subjects, RLS was associated with lower RA $(81.9 \% \pm 15.9 \%$ vs $98.5 \% \pm 29.5 \%, p=0.030)$, RAA $(84.7 \% \pm 18 \%$ vs $105.6 \% \pm 24.1 \%, p=0.022)$, LA $(109.8 \% \pm 18.6 \%$ vs $140.1 \% \pm 33.7 \%, p=0.017)$ and LAA (median $102.9 \%$ (IQR $65.6 \%-121.7 \%$ ) vs $229.1 \%$ (151.8\%-337.5\%], $p=0.002$ ) expansion indices and lower $Q p / Q$ s ratio $(0.91 \pm 0.06$ vs $0.98 \pm 0.07, p=0.027)$.

Conclusions This study suggests bi-atrial dysfunction in young adults with cryptogenic stroke, associated with moderate-to-severe RLS. Dysfunction of the atria and atrial appendages may be an additional mechanism for PF0-related stroke.

Trial registration number NCT01934725.

\section{INTRODUCTION}

The worldwide incidence of ischaemic strokes in young adults has increased in recent decades. ${ }^{1}$ Targeted secondary prevention of these strokes is of uttermost importance due

\section{Key questions}

What is already known about this subject?

- Recent studies suggest left atrial (LA) dysfunction in patients with cryptogenic stroke. LA dysfunction and blood stagnation have been suggested to be an additional contributing factor for patent foramen ovale (PF0)-related stroke.

What does this study add?

- There has been only a little interest on the right atrium in patients who had a stroke. We found that there is bi-atrial dysfunction in patients with cryptogenic ischaemic stroke, which seems to be associated with right-to-left shunt. Bi-atrial dysfunction might favour thrombus formation in the atria, atrial appendages, PF0 tunnel or aneurysmatic septum in PFO-related stroke.

How might this impact on clinical practice?

- This research gives a hint of one potential disease mechanism in cryptogenic ischaemic stroke but more research is needed to know whether secondary prevention should be changed.

to long life expectancy of young patients. Unfortunately, even half of early-onset strokes remain cryptogenic, that is, without evident causality despite thorough diagnostic evaluation. ${ }^{2-4}$

Some cryptogenic strokes are believed to originate from the heart, with patent foramen ovale (PFO) as one established source. ${ }^{2}$ However, as a good proportion of PFOs may still represent a bystander and many patients do not have a PFO, further research is needed on the mechanisms of cryptogenic strokes. ${ }^{5}$

Recent studies suggest that left atrial (LA) dynamics are altered in cryptogenic stroke. ${ }^{67}$ Atrial fibrosis and atrial cardiomyopathy have been suggested as possible mechanisms, ${ }^{89}$ but also PFO with atrial septal aneurysm has been associated with LA dysfunction, suggested to be an additional contributing factor to the mechanism of PFO-related stroke. ${ }^{10}$ However, there has been only a little interest on the right atrium (RA) in patients who 
had a stroke. Bi-atrial dysfunction and blood stagnation might contribute to thrombosis formation in patients with $\mathrm{PFO}$, as deep venous thrombosis is detected only in the minority of patients. ${ }^{11} 12$

Cardiac magnetic resonance (CMR) imaging is considered as the gold standard in assessing cardiac volumetry and volume dynamics. In this case-control study, we evaluated with CMR whether the anatomy and dynamics of RA or right atrial appendage (RAA) are different in young adults with cryptogenic stroke when comparing with stroke-free controls. An approach to estimate the RAA volume in CMR was developed, using crista terminalis and pectinate muscles as anatomical landmarks. Also, a sensitivity analysis based on right-to-left shunt (RLS) was performed.

\section{METHODS}

The data that support the findings of this study are available from the corresponding author on reasonable request. The corresponding author had full access to all the data in the study and takes responsibility for its integrity and the data analysis.

\section{Study population}

Searching for Explanations for Cryptogenic Stroke in the Young: Revealing the Etiology, Triggers and Outcome (SECRETO) is an international prospective multicentre case-control study of young adults (age 18-49) presenting with an imaging-positive first-ever ischaemic stroke of undetermined aetiology. The main study protocol has been published previously. ${ }^{13}$ Briefly, patients were included after standardised diagnostic procedures, including brain MRI, imaging of intracranial and extracranial vessels with either CT angiography or magnetic resonance angiography, and cardiac imaging to exclude established causes of ischaemic stroke. Cardiac imaging prior to study inclusion included standardised transthoracic echocardiography (TTE) and transoesophageal echocardiography (TEE) with bubble test, ${ }^{14}$ transcranial Doppler (TCD) ultrasound with bubble test, 12-lead ECG and at least 24-hour Holter ECG. Both patients with and without $\mathrm{PFO}$, irrespective of $\mathrm{PFO}$ size and associated features of interatrial septum, were included in the study. Patients were age-matched and gender-matched with stroke-free controls in a 1:1 fashion. Controls were selected at random through population registers, and in few cases patients' non-related proxies served as controls. SECRETO has been approved by the Ethics Committee of Helsinki and Uusimaa Hospital District. Written informed consent was obtained from all study participants.

In this substudy, 30 patients and 30 controls underwent CMR and new TTE with inclusion period between December 2013 and May 2017. Characteristics of study subjects have been previously published. ${ }^{615}{ }^{16}$ None of the patients had PFO closure prior to CMR.

\section{CMR protocol}

All subjects were imaged with a 1.5 - $\mathrm{T}$ Avanto ${ }^{\mathrm{fit}}$ magnetic resonance scanner (Siemens Healthcare, Erlangen, Germany) using a 32-channel receiver body coil and ECG-gating, as described previously. ${ }^{15}$ Specific technical parameters for each sequence type are given in (online supplemental table S1).

The study protocol started with half-Fourier-acquisition single-shot turbo spin-echo sequence in transaxial planes covering the entire heart. Subsequently balanced steadystate free precession cine images were acquired with cartesian sampling in 2-chamber $(\mathrm{CH})$ view, short-axis (SAX) view covering the entire heart, 3-CH, 4-CH and right-ventricular outflow tract views. Gadolinium-based contrast agent (gadoterate meglumine, Dotarem) of $0.2 \mathrm{mmol} / \mathrm{kg}$ was used to enhance images. After contrast administration, through plane phase contrast (PC) flow images were acquired for ascending aorta and pulmonary artery. Inversion time (TI) scout was used to find the most suitable TI for phase-sensitive inversion recovery. Late gadolinium enhancement (LGE) images were acquired 10-15 min after contrast administration for the entire left ventricle (LV) in SAX direction, and in 2-CH, 3-CH and 4-CH directions.

\section{CMR analysis}

All RA and RAA parameters were analysed by a single CMR-trained cardiologist (PP) and LA parameters by another CMR-trained cardiologist (JK). Other CMR parameters were analysed by CMR-trained physicians (VJ and HR). All CMR analyses were performed blinded to clinical data and case-control status. LV volumes and mass were assessed using standard protocols. ${ }^{17}$ Papillary muscles and LV outflow tract were included in the LV volume. The presence of LGE required the visibility in two orthogonal planes. Image analysis was performed using QMass MR software (V.8.1, Medis Medical Imaging Systems, Leiden, the Netherlands), except for LA volumetry, which used Segment CMR V.2.2 R7015 (Medviso AB, Lund, Sweden, http://segment.heiberg.se).$^{18}$ All measures were indexed to body surface area (BSA). ${ }^{19}$

Total pulmonary to systemic blood flow ratio (Qp/ Qs) was based on phase contrast CMR and calculated as (pulmonary stroke volume - pulmonary regurgitation volume)/(aortic stroke volume - aortic regurgitation volume).

Assessment of RA and RAA volumes at ventricular enddiastole (minimum) and end-systole (maximum) were based on manual delineation of atrial endocardial surface in each SAX slice (figures 1 and 2, online supplemental video 1). RAA was defined as the whole anterolateral triangular part of the RA characterised by endocardial pectinate muscles and demarcated by crista terminalis distinguishing it from smooth-walled venous endocardial surface. ${ }^{20}{ }^{21} \mathrm{RA}$ volume and volumetric function has earlier been studied in vitro $^{22} 23$ and in vivo ${ }^{2324}$ but to our knowledge, this delineation method has not previously been used in CMR images to estimate RAA volume. RA body volume was calculated 
A

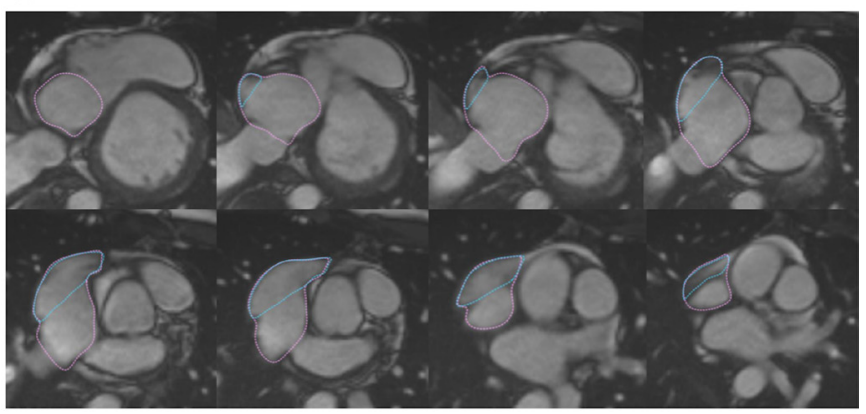

B

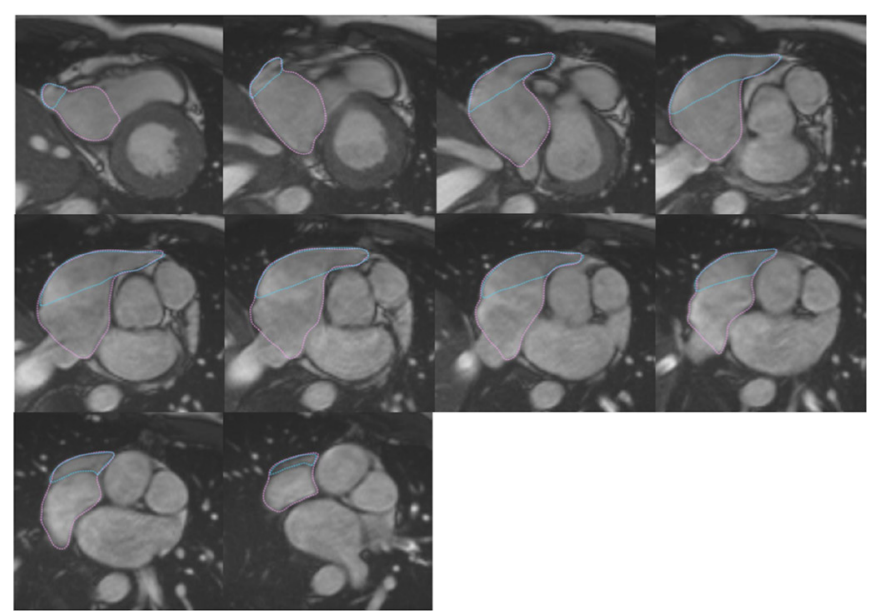

Figure 1 Delineation of right atrium (RA) and right atrial appendage (RAA) at minimum (ventricular end-diastole) (A) and maximum (ventricular end-systole) (B). RAA was defined as the whole anterolateral triangular part of the RA characterised by endocardial pectinate muscles and demarcated by crista terminalis distinguishing it from smooth-walled venous endocardial surface. ${ }^{2021}$ A patient with cryptogenic ischaemic stroke with minimal and maximal RA volumes of $56 \mathrm{~mL}$ and $112 \mathrm{~mL}$, RAA volumes of $18 \mathrm{~mL}$ and $40 \mathrm{~mL}$ and RA and RAA expansion indices of $100 \%$ and $123 \%$.

as RA volume - RAA volume. RA, RAA and RA body dynamics were described by expansion indices, calculated as (maximal volume - minimal volume) $\times 100 \% /$ minimal volume, as estimates of atrial and atrial appendage distensibility $^{2526}$ or stiffness. $^{9}$ LA and left atrial appendage (LAA) volumes and expansion indices were evaluated similarly. LAA was excluded from the LA. LA body and LAA were measured separately at ventricular end-diastole and endsystole. Atrial septal aneurysm was defined as an interatrial septal excursion $\geq 10 \mathrm{~mm}$.

\section{Echocardiography methods}

In addition to standard TTE and TEE examinations described earlier, an investigational TTE was performed by a single echocardiographist (JPi) for all study subjects blinded to the case-control status with a General Electric Vivid E9 V.113 cardiac ultrasound device, using M5Sc and $4 \mathrm{~V}$ probes (General Electric, Horten, Norway), as described previously. ${ }^{6}{ }^{16}$ TTE with Doppler was used to assess tricuspid and pulmonary valve function, peak systolic

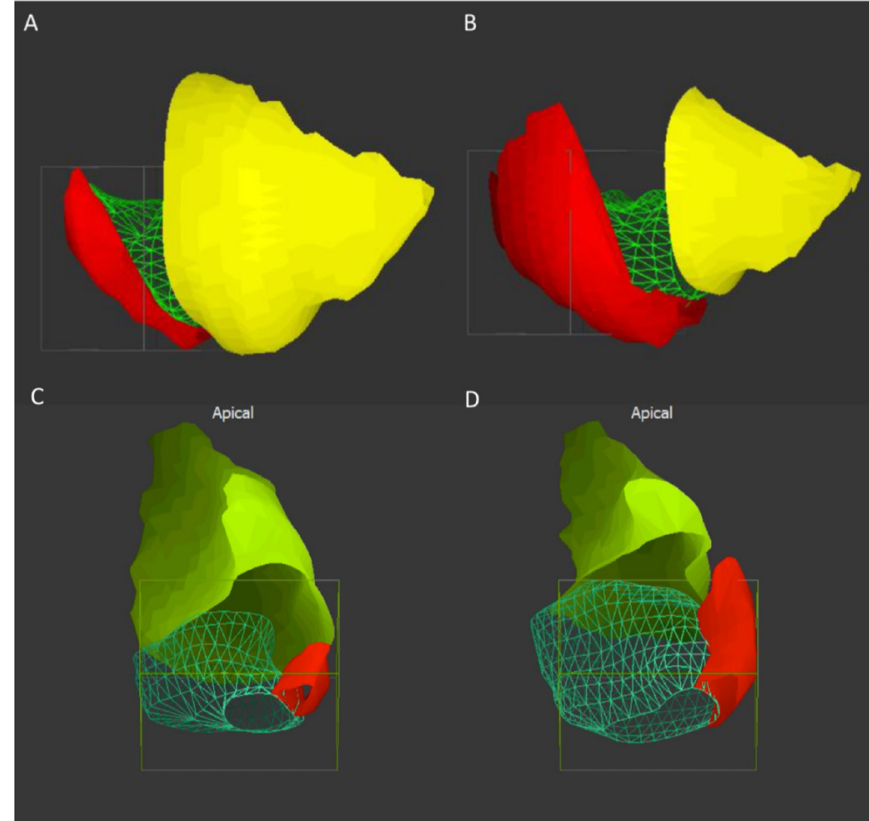

Figure 2 A lateral view of right atrial body (green), right atrial appendage (RAA) (red) and right ventricle (yellow) at ventricular end-diastole (minimum atrial volume) (A) and ventricular end-systole (maximal atrial volume) (B). Posterosuperior view of right atrial body (green), RAA (red) and right ventricle (light green) in corresponding cardiac phases (C,D). The same patient as in figure 1.

tricuspid regurgitation (TR) gradient, pulmonary artery acceleration time (PAAT) and diastolic LV function.

\section{Diagnosis of RLS}

In patients, RLS was evaluated with TEE bubble study using the standardised SECRETO echocardiography performance protocol. ${ }^{14}$ All TEE data were analysed by a single experienced cardiologist (EG) in the SECRETO echocardiography core laboratory (Bergen, Norway), blinded to clinical and CMR data, and case-control status. Agitated $10 \mathrm{~mL}$ saline-blood solution was used as a contrast and injected into the right antecubital vein. Bubble study was performed at rest and repeated during Valsalva manoeuvre in those without a severe RLS at rest. ${ }^{27}$ Moderate-to-severe RLS, defined as $\geq 10$ microbubbles appearing in the LA during the first three cardiac cycles, was considered as clinically significant. Also, the presence of LAA thrombus and atrial septal aneurysm $(\geq 10 \mathrm{~mm})$ were recorded.

In controls, RLS was evaluated with TCD bubble study (TCD-BS). TCD-BS was performed according to consensus guidelines in unilateral insonation of middle cerebral artery in supine position. ${ }^{28}$ Contrast administration and definition of RLS ( $\geq 10$ microbubbles) were similar to TEE.

\section{Statistical analysis}

Continuous variables are presented as mean $\pm \mathrm{SD}$ or median (IQR), as appropriate, and categorical variables as frequency (\%). Normality of continuous variables was tested visually and using the Shapiro-Wilk test. In case-control analyses, 
Table 1 Baseline characteristics

\begin{tabular}{|c|c|c|c|}
\hline & Patients $(n=30)$ & Controls $(n=30)$ & $P$ value \\
\hline \multicolumn{4}{|c|}{ Demographics and cardiovascular risk factors } \\
\hline Age, years & $41(35-44)$ & $42(35-45)$ & 0.131 \\
\hline Female sex & $15(50)$ & $15(50)$ & 1 \\
\hline Body surface area, $\mathrm{m}^{2}$ & $2.04 \pm 0.24$ & $1.93 \pm 0.24$ & 0.022 \\
\hline Body mass index, $\mathrm{kg} / \mathrm{m}^{2}$ & $28.4 \pm 5$ & $26.9 \pm 5.2$ & 0.294 \\
\hline Hypertension* & $9(30)$ & $4(13)$ & 0.227 \\
\hline Diabetes mellitus, type 1 & $1(3.3)$ & $0(0)$ & 1 \\
\hline Current tobacco use & $9(30)$ & $11(36.7)$ & 0.774 \\
\hline Alcohol week doses $†$ & $2(1-4)$ & $4(2-6)$ & 0.416 \\
\hline Physical inactivity & $4(13)$ & $4(13)$ & 1 \\
\hline \multicolumn{4}{|l|}{ Stroke phenotype } \\
\hline Fulfils criteria for ESUS & $21(70)$ & NA & NA \\
\hline NIH Stroke Scale & $1.5(0-4)$ & NA & NA \\
\hline Right-to-left shuntł & $7(24)$ & $1(3)$ & 0.012 \\
\hline \multicolumn{4}{|c|}{ Ventricular volumes and function-CMR } \\
\hline LV end-diastolic volume, $\mathrm{mL} / \mathrm{m}^{2}$ & 82.5 (77.9-89.3) & $84.5(76-93.5)$ & 0.910 \\
\hline LV end-systolic volume, $\mathrm{mL} / \mathrm{m}^{2}$ & $30.5 \pm 6$ & $31.1 \pm 7.2$ & 0.734 \\
\hline LV mass, $\mathrm{g} / \mathrm{m}^{2}$ & $54.8(48.5-59.9)$ & $54.8(49.1-60.2)$ & 0.417 \\
\hline LVEF, \% & $64.1 \pm 4.7$ & $63.8 \pm 5.1$ & 0.820 \\
\hline $\mathrm{RV}$ end-diastolic volume, $\mathrm{mL} / \mathrm{m}^{2}$ & $82.1(77.2-95.2)$ & $85.8(76.7-93.8)$ & 0.704 \\
\hline RV end-systolic volume, $\mathrm{mL} / \mathrm{m}^{2}$ & $34.5(28-40.7)$ & $34.5(30-40.3)$ & 0.894 \\
\hline RVEF, \% & 59.3 (54.7-63.9) & $60(57.7-63.1)$ & 0.910 \\
\hline
\end{tabular}

Values are mean $\pm \mathrm{SD}$, median (IQR) or number (\%).

Statistically significant associations $(\mathrm{P}<0.05)$ denoted in bold.

*Hypertension was based on any criteria: office hypertension $>150 / 90$, history of hypertension or blood pressure medication prior stroke. †One subject had missing data.

‡Right-to-left shunt was evaluated with transoesophageal echocardiography in patients $(n=29)$ and transcranial Doppler in controls $(n=30)-$ comparison of 29 case-control pairs.

CMR, cardiac magnetic resonance; ESUS, embolic stroke of undetermined source; LV, left ventricle; LVEF, left ventricular ejection fraction;

NA, not applicable; NIH Stroke Scale, National Institute of Health Stroke Scale; RV, right ventricle; RVEF, right ventricular ejection fraction.

comparison between paired dichotomous variables was performed with McNemar's test, paired normally distributed continuous variables with repeated measures Student's t-test and paired non-normally distributed variables with Wilcoxon signed-rank test. A sensitivity analysis based on RLS was performed using Student's t-test or Mann-Whitney $\mathrm{U}$ test, as appropriate. To study intraobserver reproducibility, Bland-Altman analysis ${ }^{29}$ was performed and intraclass correlation coefficients (ICCs) with $95 \%$ CI calculated by using a two-way mixed effects model for absolute agreement. A $p$ value of $<0.05$ was considered statistically significant and all tests were two-sided. Statistical analysis was performed on SPSS V.25 statistical package (IBM).

\section{RESULTS}

\section{Clinical characteristics}

Patients had higher BSA compared with controls (table 1). There were no differences in cardiovascular risk factors between the groups and none of the study subjects had previous cardiovascular diseases, such as coronary artery disease, atrial fibrillation, heart failure or peripheral artery disease.

Altogether 29 of 30 patients were successfully evaluated with TEE bubble study, and all 30 controls with TCD. The frequency of RLS was greater in patients (7 of 29, 24\%) compared with controls ( 1 of 30,3\%) ( $\mathrm{p}=0.012)$. Altogether five patients had an atrial septal aneurysm, of which four were accompanied with an RLS. None of the 29 patients had a LAA thrombus.

The mean duration from stroke to CMR was $31 \pm 12$ months (median 28, IQR 22-41, range 14-52) and to investigational TTE 28 12 months (median 24, IQR 16-41, range 13-48).

\section{Ventricular volumes and function}

Based on CMR, right ventricular (RV) volumes and function were similar between patients and controls, and there was no systolic RV dysfunction $(\mathrm{EF}<50 \%$ ) (table 1$)$. LV volumes, systolic function and masses were also similar, and only one patient had local LGE $(\mathrm{p}=1)$. There was no difference in $\mathrm{Qp} / \mathrm{Qs}$ shunt ratio between the groups. 
Table 2 Cardiac magnetic resonance: atrial volumes and function

\begin{tabular}{|c|c|c|c|}
\hline & Patients $(n=30)$ & Controls $(n=30)$ & $P$ value \\
\hline \multicolumn{4}{|l|}{ RA (body and appendage) } \\
\hline RA volume $\mathrm{max}, \mathrm{mL} / \mathrm{m}^{2}$ & $57.6(46.8-64.4)$ & $59.8(48.7-70.6)$ & 0.393 \\
\hline RA volume $\mathrm{min}, \mathrm{mL} / \mathrm{m}^{2}$ & $28(24.7-34.8)$ & $29.3(24.4-34.4)$ & 0.781 \\
\hline RA expansion index, \% & $86.1(68.7-114.9)$ & $95.7(73.4-123.2)$ & 0.349 \\
\hline \multicolumn{4}{|l|}{ RA body } \\
\hline RA body volume $\max , \mathrm{mL} / \mathrm{m}^{2}$ & $38.3(33.1-43.1)$ & $40(34-50.3)$ & 0.289 \\
\hline RA body volume $\mathrm{min}, \mathrm{mL} / \mathrm{m}^{2}$ & $19.6(17.3-22.6)$ & $20.1(16.3-25.7)$ & 0.441 \\
\hline RA body expansion index, \% & $93.1 \pm 31.9$ & $95.5 \pm 37.5$ & 0.759 \\
\hline \multicolumn{4}{|l|}{ RAA } \\
\hline RAA volume $\mathrm{max}, \mathrm{mL} / \mathrm{m}^{2}$ & $17.3(13.9-22.4)$ & $17.4(14.1-21.2)$ & 0.877 \\
\hline RAA volume $\mathrm{min}, \mathrm{mL} / \mathrm{m}^{2}$ & $9.3(6.7-10.5)$ & $8.2(6.3-10)$ & 0.465 \\
\hline RAA expansion index, $\%$ & $95.6 \pm 21.6$ & $108.7 \pm 25.8$ & 0.026 \\
\hline \multicolumn{4}{|l|}{ LA (body) } \\
\hline LA volume $\max , \mathrm{mL} / \mathrm{m}^{2}$ & $44.5 \pm 6.7$ & $44.7 \pm 8.3$ & 0.907 \\
\hline LA volume $\mathrm{min}, \mathrm{mL} / \mathrm{m}^{2}$ & $19.4(17.4-21.6)$ & $17.1(15-21.9)$ & 0.171 \\
\hline LA expansion index, \% & $126.2 \pm 28$ & $145 \pm 36.3$ & 0.023 \\
\hline \multicolumn{4}{|l|}{ LAA } \\
\hline LAA volume $\mathrm{max}, \mathrm{mL} / \mathrm{m}^{2}$ & $3.3(2.3-5.2)$ & $3.8(2.5-5.4)$ & 0.544 \\
\hline LAA volume $\mathrm{min}, \mathrm{mL} / \mathrm{m}^{2}$ & $1.1(0.6-2.1)$ & $1.1(0.6-2.1)$ & 0.926 \\
\hline LAA expansion index, $\%$ & $185.6(101.4-272.5)$ & $215.9(133.3-326.9)$ & 0.237 \\
\hline RAA/RA ratio at maximum, $\%$ & $31.3 \pm 4.5$ & $30.8 \pm 6.5$ & 0.764 \\
\hline $\mathrm{RAA} / \mathrm{RA}$ ratio at minimum, $\%$ & $31.1 \pm 5.1$ & $29.3 \pm 6.1$ & 0.158 \\
\hline Qp/Qs shunt ratio* & $0.96 \pm 0.08$ & $0.98 \pm 0.08$ & 0.320 \\
\hline
\end{tabular}

Values are mean \pm SD, median $(\mathrm{IQR})$ or number (\%). Expansion indices are calculated as (maximal volume - minimal volume) $\times 100 \% /$ minimal volume.

Right atrium $=$ right atrial body + right atrial appendage .

Statistically significant associations $(P<0.05)$ denoted in bold.

*One subject had missing data

LA, left atrium; LAA, left atrial appendage; Qp/Qs, the ratio of total pulmonary to total systemic blood flow; RA, right atrium; RAA, right atrial appendage.

\section{Tricuspid and pulmonary valves, pulmonary artery pressure and LV diastolic function}

Based on TTE, none of the study subjects had tricuspid or pulmonary valve disease, defined as moderate-tosevere regurgitation or stenosis. Altogether $46(77 \%)$ and $59(98 \%)$ of all study subjects had measurable peak TR gradient and PAAT. There was no difference in peak TR gradient $(19.6 \pm 2.9 \mathrm{~mm} \mathrm{Hg}$ vs $21.2 \pm 4.3 \mathrm{~mm} \mathrm{Hg} ; \mathrm{p}=0.129)$ or PAAT $(141.3 \pm 28.8 \mathrm{~ms}$ vs $145.5 \pm 27.3 \mathrm{~ms} ; \mathrm{p}=0.490)$ between patients and controls, respectively. Considering LV diastolic function, none of the study subjects had an average E/e' $>14$ with Doppler echocardiography, and all subjects had normal diastolic function according to American Society of Echocardiography and European Association of Cardiovascular Imaging criteria. ${ }^{30}$

\section{Atria and atrial appendage}

Based on CMR, atrial volumes were similar between patients and controls (table 2). RAA volume was $\sim 1 / 3$ of the total RA volume in both patients and controls (figure 2 and table 2). However, RAA expansion index $(95.6 \% \pm 21.6 \%$ vs $108.7 \% \pm 25.8 \%, \mathrm{p}=0.026)$ and LA expansion index $(126.2 \% \pm 28 \%$ vs $145 \% \pm 36.3 \%, p=0.023)$ were lower among patients compared with controls (figure 3 ).

In all 59 study subjects, RLS $(n=8)$ was associated with lower RA, RAA, LA and LAA expansion indices, and lower $\mathrm{Qp} / \mathrm{Qs}$ ratio (table 3 and figure 4), with a similar trend among patients only (online supplemental table S2).

Estimates of pulmonary pressure, PAAT or peak TR gradient, did not correlate with RA or RAA indices among patients or controls (data not shown).

\section{Repeatability of right atrial measurements}

Ten subjects were selected randomly to assess intra-observer repeatability. RA minimal and maximal volumes, and RA expansion index had ICCs of 0.991 (95\% CI: 0.962 to 0.998 , $\mathrm{p}<0.001$ ), 0.987 (95\% CI: 0.953 to $0.997, \mathrm{p}<0.001$ ) and 0.788 (95\% CI: 0.379 to $0.942, \mathrm{p}=0.001$ ), and Bland-Altman biases of $-0.1 \pm 3.4 \mathrm{~mL},-2.5 \pm 6.5 \mathrm{~mL}$ and $-7.4 \% \pm 15.4 \%$. Correspondingly, RAA minimal and maximal volumes, and RAA 
A

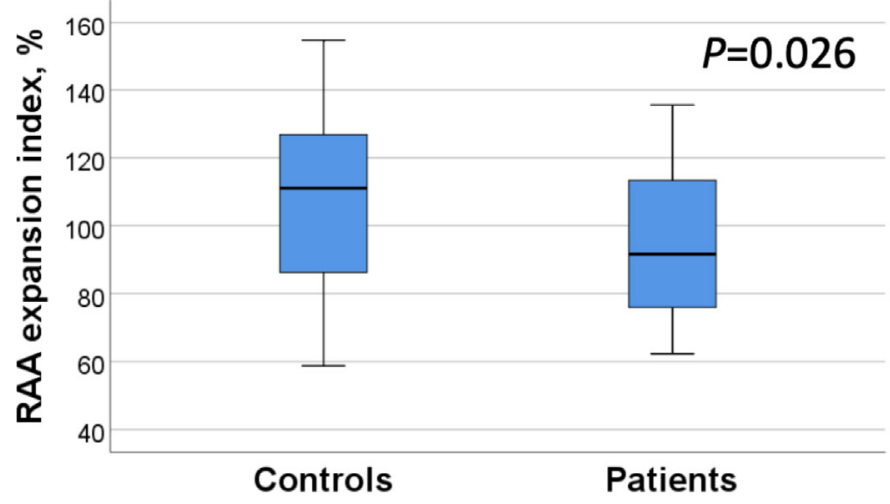

B

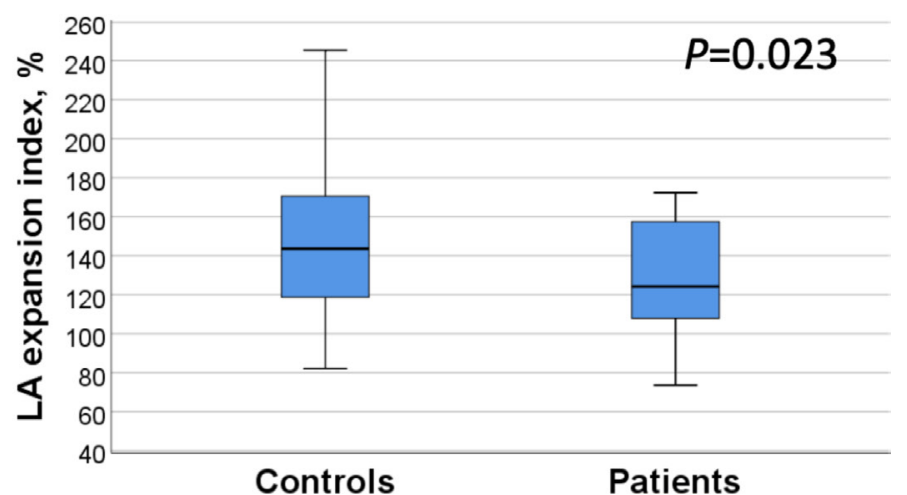

Figure 3 Right atrial appendage (RAA) (A) and left atrial (LA) (B) expansion indices in patients versus controls.

expansion index had ICCs of 0.980 (95\% CI: 0.926 to 0.995 , $\mathrm{p}<0.001), 0.979$ (95\% CI: 0.829 to $0.996, \mathrm{p}<0.001)$ and 0.789 (95\% CI: 0.389 to $0.942, \mathrm{p}=0.002$ ) with Bland-Altman biases of $-0.5 \pm 1.8 \mathrm{~mL},-2.2 \pm 2.4 \mathrm{~mL}$ and $-5.0 \% \pm 13.1 \%$.

\section{DISCUSSION}

The main finding of this study was that RAA expansion index, along with LA expansion index, is lower in young adults with cryptogenic ischaemic stroke compared with stroke-free controls. Furthermore, these indices were associated with moderate-to-severe RLS.

To our knowledge, there has not been a systematic analysis of RA involvement in cryptogenic stroke, although a concept of atrial cardiomyopathy has been presented. ${ }^{9}$ From a theoretical point of view, decreased RAA expansion in our patients could be caused by pathological

Table 3 Comparison of atrial volumes and function in all study subjects $(n=59)$ - a sensitivity analysis based on right-to-left shunt

\begin{tabular}{|c|c|c|c|}
\hline & \multicolumn{2}{|c|}{ Right-to-left shunt* } & \multirow[b]{2}{*}{$P$ value } \\
\hline & No $(n=51)$ & Yes $(n=8)$ & \\
\hline \multicolumn{4}{|l|}{ RA } \\
\hline RA volume $\mathrm{min}, \mathrm{mL} / \mathrm{m}^{2}$ & $27.7(24.3-33.8)$ & $33.7(28-35.2)$ & 0.241 \\
\hline RA expansion index, \% & $98.5 \pm 29.5$ & $81.9 \pm 15.9$ & 0.030 \\
\hline RAA volume $\max , \mathrm{mL} / \mathrm{m}^{2}$ & $17.1(13.7-21.7)$ & $18.6(16.1-24)$ & 0.341 \\
\hline RAA volume $\mathrm{min}, \mathrm{mL} / \mathrm{m}^{2}$ & $8.1(6.3-10.1)$ & $10.3(9.1-11.1)$ & 0.084 \\
\hline RAA expansion index, \% & $105.6 \pm 24.1$ & $84.7 \pm 18$ & 0.022 \\
\hline \multicolumn{4}{|l|}{ LA } \\
\hline LA volume $\max , \mathrm{mL} / \mathrm{m}^{2}$ & $44.3 \pm 7.9$ & $46.5 \pm 5.1$ & 0.452 \\
\hline \multicolumn{4}{|l|}{ LAA } \\
\hline $\mathrm{LAA}$ volume $\max , \mathrm{mL} / \mathrm{m}^{2}$ & $7.2(5.3-9.9)$ & $6(3.8-7.3)$ & 0.535 \\
\hline LAA volume $\mathrm{min}, \mathrm{mL} / \mathrm{m}^{2}$ & $2.2(1.3-3.7)$ & $2.9(1.5-4.1)$ & 0.160 \\
\hline LAA expansion index, \% & $229.1(151.8-337.5)$ & $102.9(65.6-121.7)$ & 0.002 \\
\hline Qp/Qs† & $0.98 \pm 0.07$ & $0.91 \pm 0.06$ & 0.027 \\
\hline
\end{tabular}

Values are mean \pm SD or median (IQR). Expansion indices are calculated as (maximal volume-minimal volume) x 100\%/minimal volume. Pvalues, see Statistics section for sensitivity analysis.

Statistically significant associations $(\mathrm{P}<0.05)$ denoted in bold.

*Shunt defined as moderate-to-severe and evaluated with transoesophageal echocardiography in patients $(n=29)$ and transcranial Doppler in controls $(n=30)$.

†One subject had missing data.

LA, left atrium; LAA, left atrial appendage; Qp/Qs, the ratio of total pulmonary to total systemic blood flow; RA, right atrium; RAA, right atrial appendage. 
A

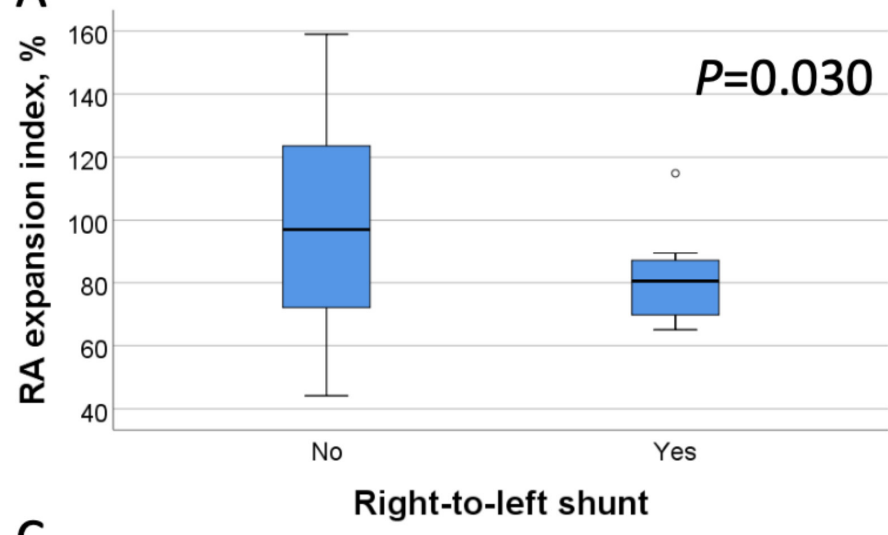

C

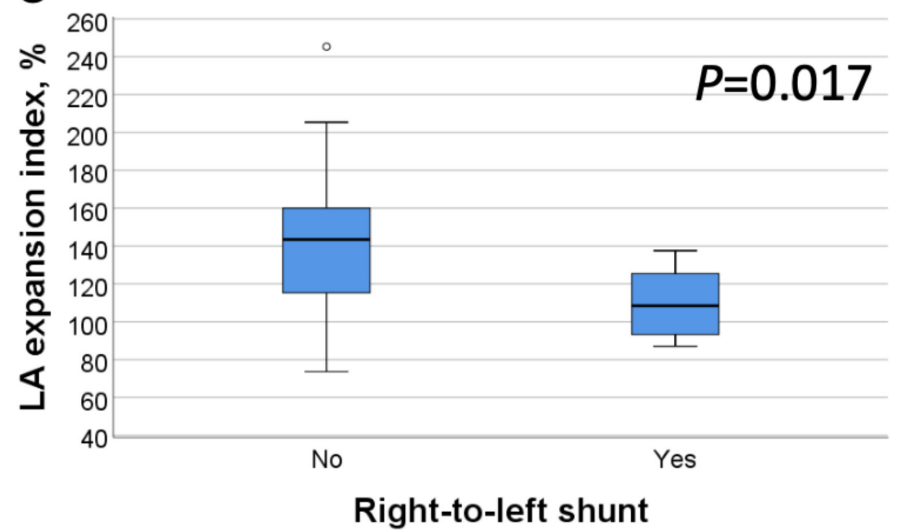

B

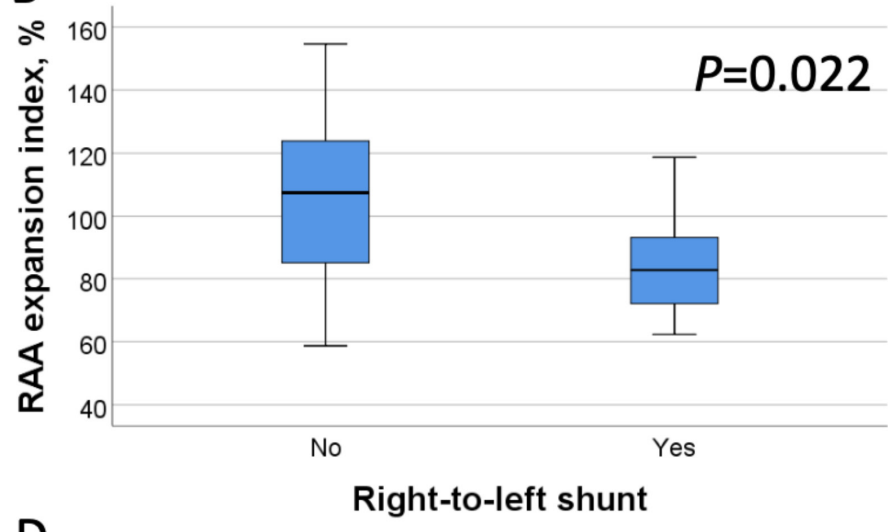

D

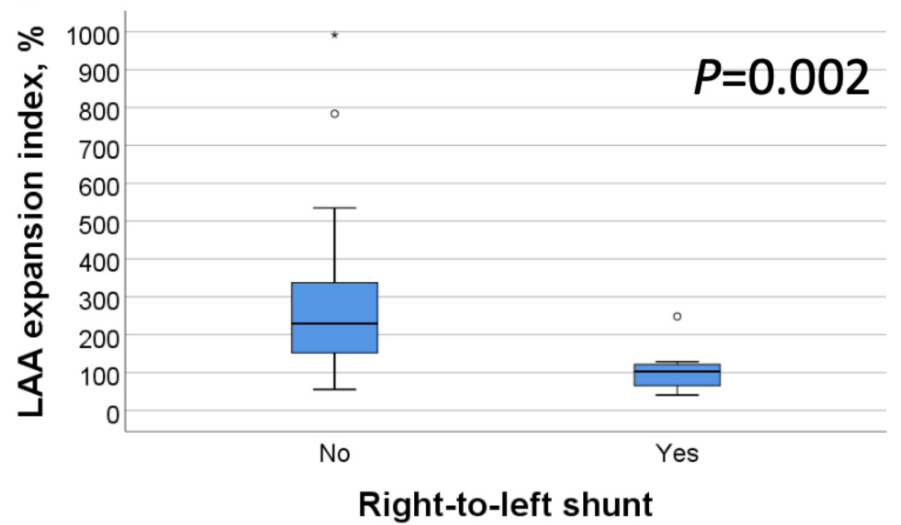

Figure 4 Comparison of right atrial (RA), right atrial appendage (RAA), left atrial (LA) and left atrial appendage (LAA) expansion indices in all study subjects, based on right-to-left shunt.

loading conditions, processes affecting primarily the atria or both. We found no evidence of increased loading conditions, as there were no tricuspid or pulmonary valve diseases, elevated pulmonary artery pressure or RV systolic dysfunction in our patients, neither was there LV dysfunction. However, $24 \%$ of our patients had a moderate-tosevere RLS, compared with only $3 \%$ of controls. Notably, RLS was associated with bi-atrial dysfunction among all study subjects, that is, lower RA, RAA, LA and LAA expansion indices, and also with lower Qp/Qs ratio, strengthening the idea that significant shunting really occurs in these subjects.

Decreased LA expansion in our patients is in accordance with an earlier study, in which PFO with atrial septal aneurysm was associated with LA dysfunction. ${ }^{10}$ In that study, authors presented that 'atrial fibrillation-like' LA dysfunction, which normalised after PFO closure, might be an additional contributing mechanism for stroke in patient with PFO. In a previous computational flow dynamics study, severe permanent RLS without atrial septal aneurysm was associated with blood stagnation across the LA, and especially across the LAA, suggesting reduction of atrial contraction in these patients with similar thrombogenic pathophysiology as in atrial fibrillation. ${ }^{31}$ Recently, LA diameter was associated with the clinical severity of PFO, further suggesting PFO-associated LA cardiomyopathy. ${ }^{32}$ Similarly, altered RA, RAA, LA and
LAA dynamics in our patients might lead to intra-atrial conditions that favour thrombus formation in the atria, atrial appendages, PFO tunnel or aneurysmatic atrial septum. Atrial involvement in the pathogenesis of cryptogenic strokes might explain why a broad range of patients with PFO-related stroke benefit from PFO closure in secondary prevention, ${ }^{33}$ despite the fact that deep venous thrombosis is detected only in a minority of patients. ${ }^{11} 12$

One possible explanation for RLS-associated RA dysfunction in our study subjects is that interatrial shunting flattens RA pressure and volume changes. Also, the RAA might be more sensitive to pressure changes than RA body, as the entire RAA is covered by pectinate muscles and the wall between these muscle ridges is thin and almost parchment-like. ${ }^{20}$ On the other hand, RA or RAA stiffness might also assist the opening of RLS and increase the size of shunting. Considering LA dysfunction in our study subjects with RLS, similar impairment of active and passive LA emptying, and conduit function, as previously demonstrated is probable. ${ }^{10}$

RA volumes in our study subjects were similar to earlier CMR reference values. ${ }^{34}$ The mean RA emptying fraction in our controls $(49 \% \pm 7 \%)$ was comparable with normal reference values of Sievers et al $(47 \% \pm 8 \%)$ and Maceira et al $(56 \% \pm 7 \%) .{ }^{24}{ }^{35}$ In our study, the mean ratio of maximal RA to LA volume in control subjects was similar $(1.4 \pm 0.3)$ to that reported earlier. ${ }^{23}$ There is very limited 
information on the RAA function in healthy individuals. We developed an approach to estimate the RAA volume in CMR images, using landmarks of crista terminalis and pectinate muscles, ${ }^{20}{ }^{21}$ with a good intra-observer repeatability. In our patients and controls, the estimated RAA volume was $\sim 1 / 3$ of the total RA volume at minimum (ventricular end-diastole) and maximum (ventricular end-systole).

\section{Strengths and limitations}

As a major strength, our study was based on prospectively enrolled participants with thorough baseline characterisation for both patients and controls, including structural and standardised echocardiography protocols, TCD-BS and comprehensive CMR protocol. ${ }^{13}{ }^{14}$ All TEE data were analysed separately in the central core laboratory, blinded to CMR analysis. A limitation of this study is that the number of participants was relatively modest, which restricted possibilities to adjust for confounders, although we matched controls for age and gender, indexed parameters for BSA and performed a sensitivity analysis with RLS. Thus, our findings should be treated as hypothesis generating and verified in a larger patient cohort. Also, we had no invasive RV or LV filling pressure measurements. Furthermore, it must be reminded that only a portion of patients with PFO have moderate-tosevere RLS, and mild shunts probably have smaller effect on atrial function. Also, our data sample was too small to compare atrial function in groups of patients with severe or permanent shunt to those with moderate shunt. In future CMR studies, atrial fibrosis detected in older patients who had a stroke with undetermined cause, ${ }^{89}$ should be assessed as a possible contributor for the atrial function in young adults with cryptogenic stroke.

\section{CONCLUSIONS}

This study suggests bi-atrial dysfunction in young adults with cryptogenic stroke, associated with moderate-tosevere RLS. Dysfunction of the atria and atrial appendages may be an additional mechanism for PFO-related stroke.

\section{Acknowledgements We are indebted to Laura-Leena Kupari, RN, for her contributions throughout the study.}

Contributors PP: data curation, image and data analysis, validation, writing the manuscript and editing; JK: image analysis, editing the manuscript; JPi: performing cardiac magnetic resonance and echocardiography, echocardiography image analysis, editing the manuscript; HR: image analysis, editing the manuscript; LL: planning cardiac MRI protocols, editing the manuscript; RP: investigation, editing the manuscript; NM-M: obtaining data, data curation, writing and editing the manuscript; EG: transoesophageal echocardiography analysis, editing the manuscript; JPu: planning the study, obtaining data, supervision, funding, editing the manuscript; JS: planning the study, supervision, editing the manuscript; VJ: planning the study, planning and performing imaging protocols, image analysis, supervision, editing the manuscript.

Funding This study has received funding by the Hospital District of Helsinki and Uusimaa (TYH2018318) and Academy of Finland (286246 and 318075). JPi reports research grants from Finska Läkaresällskapet, Maire Taposen Säätio,, Aarne Koskelon Säätiö and Stiftelsen Dorothea Olivia, Karl Walter och Jarl Walter Perkléns Minne, during the conduct of the study.
Competing interests JPi reports personal fees from General Electric Healthcare, outside the submitted work. JPu reports personal fees from Boehringer Ingelheim, Bayer, Portola and Terve Media, research grants and personal fees from BMS-Pfizer and Abbott/St Jude Medical, research grants from Business Finland and Amgen, research collaboration with Nokia Technologies, Bittium and BcB Medical, research collaboration and stock ownership with Vital Signum, outside the submitted work; and he has participated in the European Stroke Organisation's guideline working groups on (1) Post-stroke hyperglycaemia and (2) Secondary prevention in patients with atrial fibrillation, and in the Finnish Duodecim Society's guideline working group on ischaemic stroke and transient ischaemic attack. Other authors have nothing to disclose.

Patient consent for publication Not required.

Provenance and peer review Not commissioned; externally peer reviewed.

Data availability statement Data are available upon reasonable request.

Open access This is an open access article distributed in accordance with the Creative Commons Attribution Non Commercial (CC BY-NC 4.0) license, which permits others to distribute, remix, adapt, build upon this work non-commercially, and license their derivative works on different terms, provided the original work is properly cited, appropriate credit is given, any changes made indicated, and the use is non-commercial. See: http://creativecommons.org/licenses/by-nc/4.0/.

ORCID iD

Pauli Pöyhönen http://orcid.org/0000-0002-3031-5391

\section{REFERENCES}

1 Béjot Y, Delpont B, Giroud M. Rising stroke incidence in young adults: more epidemiological evidence, more questions to be answered. J Am Heart Assoc 2016;5. doi:10.1161/JAHA.116.003661. [Epub ahead of print: 11 May 2016].

2 Yesilot Barlas N, Putaala J, Waje-Andreassen U, et al. Etiology of first-ever ischaemic stroke in European young adults: the 15 cities young stroke study. Eur J Neurol 2013;20:1431-9.

3 Rolfs A, Fazekas F, Grittner U, et al. acute cerebrovascular disease in the young: the stroke in young Fabry patients study. Stroke 2013;44:340-9.

4 Li L, Yiin GS, Geraghty OC, et al. Incidence, outcome, risk factors, and long-term prognosis of cryptogenic transient ischaemic attack and ischaemic stroke: a population-based study. Lancet Neurol 2015;14:903-13.

5 Pristipino C, Sievert H, D'Ascenzo F, et al. European position paper on the management of patients with patent foramen ovale. General approach and left circulation thromboembolism. Eur Heart $J$ 2019;40:3182-95.

6 Pirinen J, Järvinen V, Martinez-Majander N, et al. Left atrial dynamics is altered in young adults with cryptogenic ischemic stroke: a casecontrol study utilizing advanced echocardiography. J Am Heart Assoc 2020;9:e014578.

7 Meisel K, Yuan K, Fang Q, et al. Embolic stroke of undetermined source: a population with left atrial dysfunction. $J$ Stroke Cerebrovasc Dis 2019;28:1891-6.

8 Fonseca AC, Alves P, Inácio N, et al. Patients with undetermined stroke have increased atrial fibrosis: a cardiac magnetic resonance imaging study. Stroke 2018;49:734-7.

9 Goette A, Kalman JM, Aguinaga L, et al. EHRA/HRS/APHRS/ SOLAECE expert consensus on atrial cardiomyopathies: definition, characterization, and clinical implication. Europace 2016;18:1455-90.

10 Rigatelli G, Aggio S, Cardaioli P, et al. Left atrial dysfunction in patients with patent foramen ovale and atrial septal aneurysm: an alternative concurrent mechanism for arterial embolism? JACC Cardiovasc Interv 2009;2:655-62.

11 Lethen H, Flachskampf FA, Schneider R, et al. Frequency of deep vein thrombosis in patients with patent foramen ovale and ischemic stroke or transient ischemic attack. Am J Cardiol 1997;80:1066-9.

12 Lapergue B, Decroix JP, Evrard S, et al. Diagnostic yield of venous thrombosis and pulmonary embolism by combined CT venography and pulmonary angiography in patients with cryptogenic stroke and patent foramen ovale. Eur Neurol 2015;74:69-72.

13 Putaala J, Martinez-Majander N, Saeed S, et al. Searching for explanations for cryptogenic stroke in the young: revealing the triggers, causes, and outcome (SECRETO): rationale and design. Eur Stroke J 2017;2:116-25.

14 Saeed S, Gerdts E, Waje-Andreassen U, et al. Searching for explanations for cryptogenic stroke in the young: revealing the 
etiology, triggers, and outcome (SECRETO): echocardiography performance protocol. Echo Res Pract 2019;6:53-61.

15 Pöyhönen P, Kuusisto J, Järvinen V, et al. Left ventricular noncompaction as a potential source for cryptogenic ischemic stroke in the young: a case-control study. PLoS One 2020;15:e0237228.

16 Pirinen J, Kuusisto J, Järvinen V, et al. Diastolic function in young patients with cryptogenic stroke: a case-control pilot study. Clin Physiol Funct Imaging 2020;40:336-42.

17 Schulz-Menger J, Bluemke DA, Bremerich J, et al. Standardized image interpretation and post processing in cardiovascular magnetic resonance: Society for cardiovascular magnetic resonance (SCMR) board of trustees Task force on standardized post processing. $J$ Cardiovasc Magn Reson 2013;15:35.

18 Heiberg E, Sjögren J, Ugander M, et al. Design and validation of Segment--freely available software for cardiovascular image analysis. BMC Med Imaging 2010;10:1. - .

19 Mosteller RD. Simplified calculation of body-surface area. N Engl J Med 1987;317:1098.

20 Ho SY, Anderson RH, Sánchez-Quintana D. Atrial structure and fibres: morphologic bases of atrial conduction. Cardiovasc Res 2002;54:325-36.

21 Anderson $\mathrm{RH}$, Cook AC. The structure and components of the atrial chambers. Europace 2007;9(Suppl 6):vi3-9.

22 Jauhiainen T, Järvinen VM, Hekali PE, et al. MR gradient echo volumetric analysis of human cardiac casts: focus on the right ventricle. J Comput Assist Tomogr 1998;22:899-903.

23 Järvinen VM, Kupari MM, Hekali PE, et al. Right atrial MR imaging studies of cadaveric atrial casts and comparison with right and left atrial volumes and function in healthy subjects. Radiology 1994;191:137-42.

24 Sievers B, Addo M, Breuckmann F, et al. Reference right atrial function determined by steady-state free precession cardiovascular magnetic resonance. J Cardiovasc Magn Reson 2007:9:807-14

25 Hsiao S-H, Chiou K-R, Lin K-L, et al. Left atrial distensibility and $\mathrm{E} / \mathrm{e}^{\prime}$ for estimating left ventricular filling pressure in patients with stable angina. -A comparative echocardiography and catheterization study-. Circ J 2011;75:1942-50.
26 Hsiao S-H, Chu K-A, Wu C-J, et al. Left atrial expansion index predicts left ventricular filling pressure and adverse events in acute heart failure with severe left ventricular dysfunction. J Card Fail 2016;22:272-9.

27 Silvestry FE, Cohen MS, Armsby LB, et al. Guidelines for the echocardiographic assessment of atrial septal defect and patent foramen ovale: from the American Society of echocardiography and Society for cardiac angiography and interventions. J Am Soc Echocardiogr 2015;28:910-58.

28 Tsivgoulis G, Stamboulis E, Sharma VK, et al. Safety of transcranial Doppler 'bubble study' for identification of right to left shunts: an international multicentre study. J Neurol Neurosurg Psychiatry 2011;82:1206-8.

29 Bland JM, Altman DG. Statistical methods for assessing agreement between two methods of clinical measurement. Lancet 1986;1:307-10.

30 Nagueh SF, Smiseth OA, Appleton CP, et al. Recommendations for the evaluation of left ventricular diastolic function by echocardiography: an update from the American Society of echocardiography and the European association of cardiovascular imaging. Eur Heart J Cardiovasc Imaging 2016;17:1321-60.

31 Rigatelli G, Zuin M, Fong A. Computational flow dynamic analysis of right and left atria in patent foramen ovale: potential links with atrial fibrillation. J Atr Fibrillation 2018;10:10.

32 Rigatelli G, Zuin M, Adami A, et al. Left atrial enlargement as a maker of significant high-risk patent foramen ovale. Int $J$ Cardiovasc Imaging 2019;35:2049-56.

33 Turc G, Calvet D, Guérin P, et al. Closure, anticoagulation, or antiplatelet therapy for cryptogenic stroke with patent foramen ovale: systematic review of randomized trials, sequential meta-analysis, and new insights from the close study. J Am Heart Assoc 2018;7.

34 Maceira AM, Cosín-Sales J, Roughton M, et al. Reference right atrial dimensions and volume estimation by steady state free precession cardiovascular magnetic resonance. J Cardiovasc Magn Reson 2013;15:29

35 Maceira AM, Cosin-Sales J, Prasad SK, et al. Characterization of left and right atrial function in healthy volunteers by cardiovascular magnetic resonance. J Cardiovasc Magn Reson 2016;18:64-8. 